\title{
Percolation transition and the onset of nonexponential relaxation in fully frustrated models
}

\author{
Annalisa Fierro, Giancarlo Franzese, Antonio de Candia, and Antonio Coniglio \\ Dipartimento di Scienze Fisiche, Università Degli Studi di Napoli “Federico II,'” Mostra d'Oltremare, \\ Padiglione 19, 80125 Napoli, Italy \\ and INFM, Unità di Napoli, Napoli, Italy \\ (Received 11 March 1998; revised manuscript received 30 July 1998)
}

\begin{abstract}
We numerically study the dynamical properties of fully frustrated models in two and three dimensions. The results obtained support the hypothesis that the percolation transition of the Kasteleyn-Fortuin clusters corresponds to the onset of stretched exponential autocorrelation functions in systems without disorder. This dynamical behavior may be due to the "large scale" effects of frustration, present below the percolation threshold. Moreover, these results are consistent with the picture suggested by Campbell et al. [J. Phys. C 20, L47 (1987)] in the space of configurations. [S1063-651X(98)07412-1]

PACS number(s): $05.50 .+\mathrm{q}$
\end{abstract}

\section{INTRODUCTION}

At low temperature, spin glasses (SG's) undergo a transition characterized by the divergence of the nonlinear susceptibility. Moreover, the relaxation functions of the system become nonexponential at temperatures higher than the transition temperature $T_{s g}$. This behavior has been observed in canonical metallic and insulating spin glasses, investigated by neutron and hyperfine techniques [1].

In the Ising SG model, studied with spin-flip Monte Carlo dynamics, both in two [2] and three dimensions [3], nonexponential relaxation functions have been observed below some temperature $T^{*}$ higher than $T_{s g}$. Moreover in the three-dimensional (3D) system Ogielski [3] observed that the long time regime of the relaxation functions is well approximated by the following function:

$$
f(t)=f_{0} t^{-x} \exp \left[-(t / \tau)^{\beta}\right]
$$

Fitting the data with this function, he obtained that the onset of nonexponential relaxation is consistent with the Griffiths temperature $T_{G}$, that coincides with the critical temperature of the ferromagnetic model.

This result supports the argument suggested in Ref. [4], recently verified by more rigorous analysis in Ref. [5], and by numerical simulations on a generalization of the SG model in Refs. $[7,8]$. According to these analyses, in the SG the onset $T^{*}$ of the nonexponential relaxation should be greater than or equal to the Griffiths temperature $T_{G}$. This behavior is caused by the existence of unfrustrated ferromagnetic-type clusters of interactions, the same as those responsible for the Griffiths singularity [6]. The presence of nonexponential relaxation in this approach is therefore a direct consequence of the quenched disorder.

Another mechanism leading to nonexponential relaxation in frustrated systems, such as SG's, has been suggested by several authors [9-11]. According to these arguments the onset $T^{*}$ of nonexponential relaxation is greater than or equal to the percolation transition $T_{p}$ of the KasteleynFortuin and Coniglio-Klein (KFCK) clusters [12,13]. How- ever, in frustrated systems with disorder, $T_{p}$ is less than but close to $T_{G}$; therefore its eventual effects are hidden by those related to $T_{G}$.

A way to verify if percolation mechanisms can play a role in the dynamical transition of frustrated systems is to consider frustrated models without disorder, where the Griffiths phase does not exist. In particular, we have considered fully frustrated (FF) spin systems [14], where ferromagnetic and antiferromagnetic interactions are distributed in a regular way on the lattice, in such a way that no unfrustrated region (no Griffiths phase) exists, but the percolation temperature of KFCK clusters is still defined.

In a previous paper [7] we studied the 2D FF Ising model. We found numerically that the model exhibits a nonexponential relaxation below the percolation temperature $T_{p}$ of the KFCK clusters. Moreover the long time regime of these functions is well approximated by a Kohlrausch-WilliamsWatts function, also known as the "stretched exponential"

$$
f(t)=f_{0} \exp \left[-(t / \tau)^{\beta}\right]
$$

In this paper we analyze, with conventional spin flip, the dynamical behavior of the FF Ising model in three and two dimensions using better statistics, finding that $T^{*}$ is numerically consistent with $T_{p}$.

To clarify the role of percolation we study also the 3D $q$-bond FF percolation model. For $q=2$ this model is obtained applying the KFCK cluster formalism to the FF Ising model (see Sec. II). We simulate it using the "bond flip" dynamics [7]. In this way the percolation properties of the model are stressed, and the appearance of nonexponential relaxation functions at $T_{p}$ are more evident.

In both these cases we find that the relaxation functions exhibit an exponential long time behavior at high temperatures. Below the percolation temperature $T_{p}$ of the KFCK clusters, which is higher than the transition temperature $T_{c}$ of the model, the long time regime of the relaxation functions becomes nonexponential and is well approximated by a stretched exponential. Our results are consistent with the picture suggested by Campbell et al. [9] in the space of configu- 


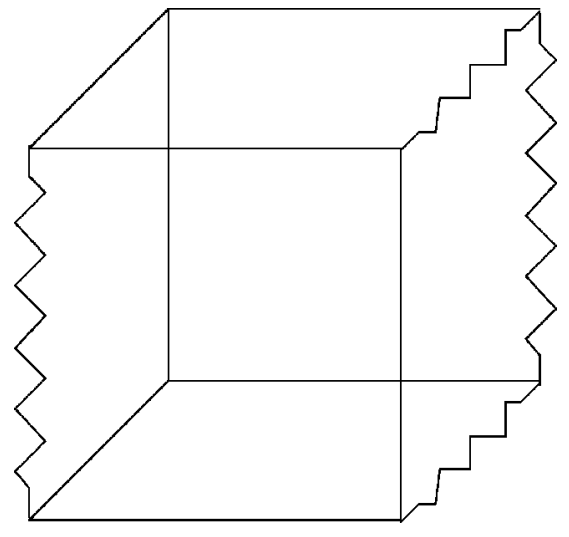

FIG. 1. Distribution of interactions for the FF model. Straight lines and wavy lines correspond, respectively, to $\epsilon_{i j}=1$ and -1 .

rations, and can be interpreted considering that $T_{p}$ corresponds to a thermodynamic transition in a generalized frustrated model [8].

In Sec. II we present the " $q$-bond frustrated percolation" model, and in Sec. III we study the percolation properties of this model on a FF cubic lattice. We find that the percolation transition is in the same universality class of the $q / 2$-state ferromagnetic Potts model, confirming the results obtained in the disordered version of the model in 2D [8]. In Sec. IV we study the FF Ising model dynamical properties with conventional spin flip, and in Sec. V we present the relaxation functions obtained simulating the FF $q$-bond percolation model for $q=2$, with the "bond flip" dynamics. In Sec. VI we show the connection with the Campbell scenario [9], and in Sec. VII we give conclusions.

\section{II. “ $q$-BOND FRUSTRATED PERCOLATION” MODEL}

The FF Ising spin model is defined by the Hamiltonian

$$
\mathcal{H}=-J \sum_{\langle i j\rangle}\left(\epsilon_{i j} S_{i} S_{j}-1\right)
$$

where $\epsilon_{i j}$ are quenched variables which assume the values \pm 1 . The ferromagnetic and antiferromagnetic interactions are distributed in a regular way on the lattice (see Fig. 1).

Using the KFCK cluster formalism for frustrated spin Hamiltonians [15], it is possible to show that the partition function of the model Hamiltonian in Eq. (3) is given by

$$
Z=\sum_{C}^{*} e^{\mu n(C) / k_{B} T} q^{N(C)},
$$

where $q=2$ is the multiplicity of the spins, $k_{B}$ is the Boltzmann constant, $\mu=k_{B} T \ln \left(e^{q J / k_{B} T}-1\right)$, and $n(C)$ and $N(C)$, respectively, are the number of bonds and the number of clusters in the bond configuration $C$. The summation $\Sigma_{C}^{*}$ extends over all the bond configurations that do not contain a "frustrated loop," that is, a closed path of bonds which contains an odd number of antiferromagnetic interactions. Note that there is only one parameter in the model, namely, the temperature $T$, ranging from 0 to $\infty$. The parameter $\mu$, that can assume positive or negative values, plays the role of a chemical potential.
Varying $q$, we obtain an entire class of models differing by the "multiplicity" of the spins, which we call the $q$-bond FF percolation model. More precisely, for a general value of $q$, the model can be obtained from a Hamiltonian [16]

$$
\mathcal{H}=-s J \sum_{\langle i j\rangle}\left[\left(\epsilon_{i j} S_{i} S_{j}+1\right) \delta_{\sigma_{i} \sigma_{j}}-2\right],
$$

in which every site carries two types of spin, namely, an Ising spin and a Potts spin $\sigma_{i}=1, \ldots, s$ with $s=q / 2$. For $q$ $=1$ the factor $q^{N(C)}$ disappears from Eq. (4), and we obtain a simpler model in which the bonds are randomly distributed under the conditions that the bond configurations do not contain a frustrated loop. For $q \rightarrow 0$ we recover the tree percolation, in which all loops are forbidden, be they frustrated or not [17].

When all the interactions are positive (i.e., $\epsilon_{i j}=1$ ) the sum in Eq. (4) contains all bond configurations without any restriction. In this case the partition function coincides with the partition function of the ferromagnetic $q$-state Potts model, which in the limit $q=1$ gives the random bond percolation [17].

From renormalization group [18], mean field [19] and numerical results $[8,20]$, we expect that the model in Eq. (5) exhibits two critical points: the first at a temperature $T_{p}(q)$, corresponding to the percolation of the bonds on the lattice, in the same universality class of the ferromagnetic $q / 2$-state Potts model; the other at a lower temperature $T_{c}(q)$, in the same universality class as the FF Ising model.

\section{STATIC PROPERTIES}

In this section we analyze the percolation properties of the model defined by Eq. (5) for $q=2$, on a FF cubic lattice. After preliminary runs with spin-flip dynamics on systems with lattice sizes $L=10$ and 20 , and with statistics of $5 \times 10^{3}$ thermalization steps and $5 \times 10^{6}$ acquisition steps, we found that the percolation transition occurs well above the critical temperature $T_{c}=1.35$ [21] (in the following the temperatures will be given in $J / k_{B}$ units). Then we simulated the model for $L=30-80$, by Swendsen-Wang cluster dynamics [22], that turns out to be very efficient for the temperature regime of interest, allowing one to consider only $5 \times 10^{4}$ acquisition steps. At every step we evaluated the percolation probability

$$
P=1-\sum_{s} s n_{s}
$$

and the mean cluster size

$$
S=\sum_{s} s^{2} n_{s}
$$

where $n_{s}$ is the density of finite clusters of size $s$.

Around the percolation temperature, the averaged quantities $P(T)$ and $S(T)$, for different values of the lattice size $L$, should obey to the finite size scalings [23]

$$
\begin{gathered}
P(T)=L^{-\beta / \nu} F_{P}\left[L^{1 / \nu}\left(T-T_{p}\right)\right], \\
S(T)=L^{\gamma / \nu} F_{S}\left[L^{1 / \nu}\left(T-T_{p}\right)\right],
\end{gathered}
$$



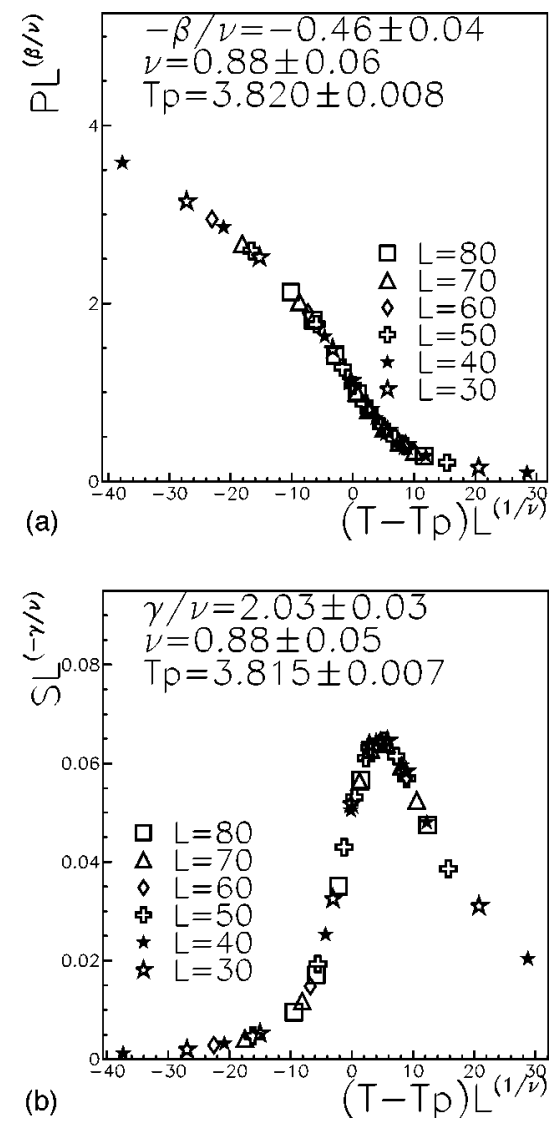

FIG. 2. Finite size scaling of (a) $P(T)$ and (b) $S(T)$, for the $q$ $=2$ model, and for lattice sizes $L=30,40,50,60,70$, and 80 .

where $\beta, \gamma$, and $\nu$ are critical exponents, and $F_{P}(x)$ and $F_{S}(x)$ are universal functions of an adimensional quantity $x$. Standard scaling analysis results are summarized in Fig. 2. We obtained $T_{p}=3.817 \pm 0.005, \nu=0.88 \pm 0.06, \beta / \nu=0.46$ \pm 0.04 , and $\gamma / \nu=2.03 \pm 0.03$. The values of the critical exponents coincide, within the errors, with the random bond percolation exponents [23]. As we expect, the $q=2$ bond frustrated percolation model is in the same universality class of the $q / 2=1$ state ferromagnetic Potts model.

\section{RELAXATION FUNCTIONS OF THE FULLY FRUSTRATED ISING SPIN MODEL}

In this section we present our results in the study of the FF Ising model, defined by the Hamiltonian in Eq. (5) for $q=2$, simulated by spin-flip dynamics. For each temperature $T, 16$ different runs were made, varying the random number generator seed, on a FF cubic lattice of size $L=30$. We took about $10^{4}$ steps for thermalization, and about $10^{5}$ steps for acquisition, calculating at each step the energy $E(t)$. The relaxation function of the energy is defined as

$$
f(t)=\frac{\langle\delta E(t) \delta E(0)\rangle}{\left\langle(\delta E)^{2}\right\rangle},
$$

where $\delta E(t)=E(t)-\langle E\rangle$. For each value of $T$, we averaged the 16 functions calculated and evaluated the error as a stan-

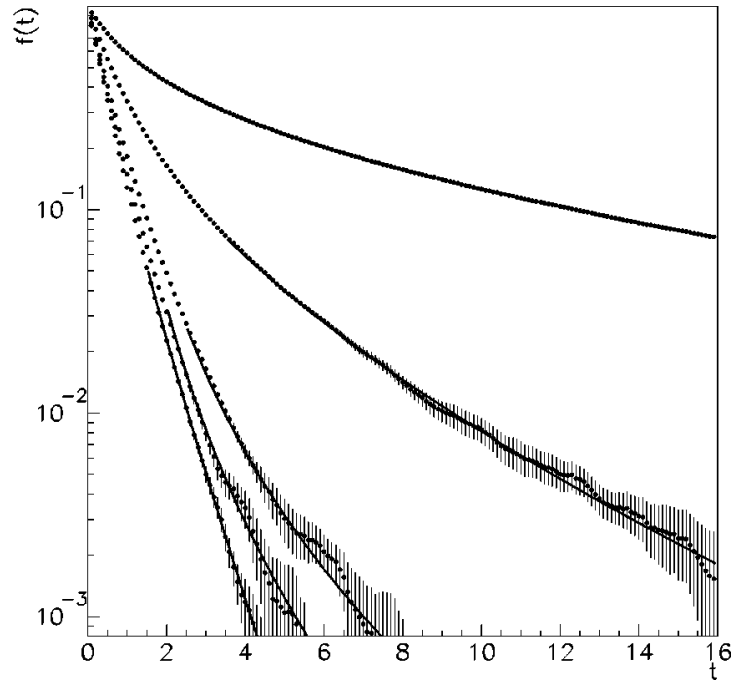

FIG. 3. Relaxation functions $f(t)$ of energy as a function of time $t$ for the $d=3$ FF Ising model, with spin flip dynamics and lattice size $L=30$, for temperatures (from left to right) $T=4.0,3.5,3.0$, 2.0, and 1.5.

dard deviation of the mean. Here a unit of time is considered to be one Monte Carlo step, that is $L^{d}$ single spin update trials.

In Fig. 3 we show the results for $T=4.0,3.5,3.0,2.0$, and 1.5. We also observe a two step decay for high temperatures. For all the temperatures we fit the long time tail of the relaxation functions with the empirical formula proposed in Eq. (1) by Ogielski.

The temperature dependence of exponents $\beta(T)$ is presented in Fig. 4. Note that $\beta(T)$ increases as function of $T$ from the value $\beta=0.58 \pm 0.03$ for $T=1.5$ to the value $\beta$ $=1$ for $T=3.7$ and 4.0. We do not observe any regular behavior in the temperature dependence of exponent $x(T)$. We estimated the errors on parameters as the range where we obtain a good fit of the relaxation function. As we can see in Fig. 4, these results are consistent, within the errors, with the

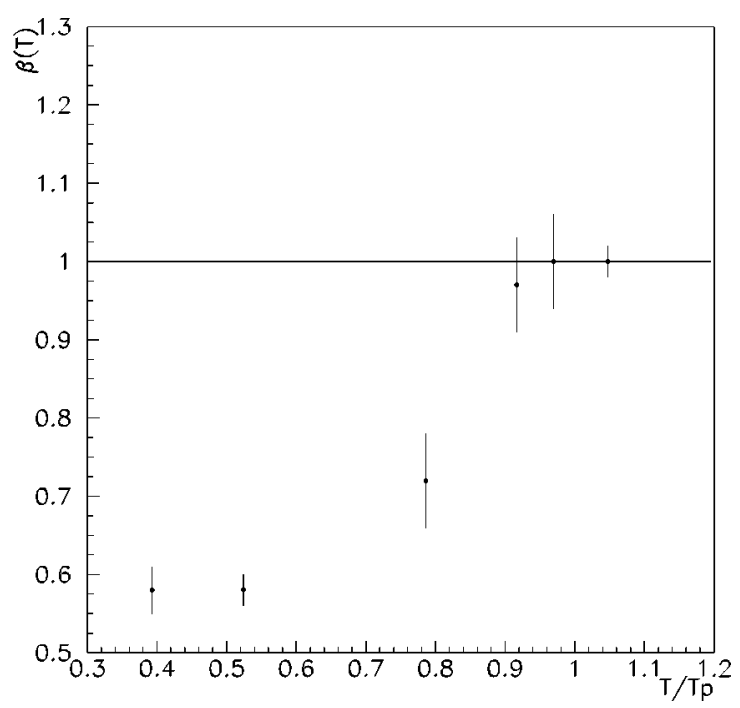

FIG. 4. Stretching exponents $\beta(T)$ as a function of $T / T_{p}$, the ratio of temperature over percolation temperature, for the $d=3 \mathrm{FF}$ Ising model, with spin flip dynamics and lattice size $L=30$. 


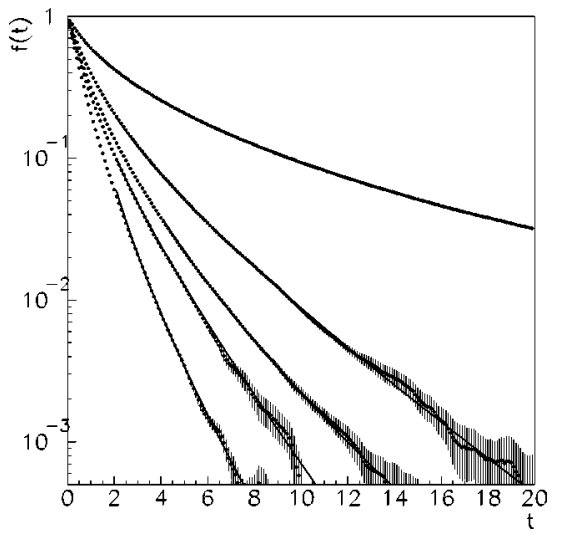

FIG. 5. Relaxation functions $f(t)$ of energy as a function of time $t$ for the $d=2$ FF Ising model, with spin flip dynamics and lattice size $L=60$, for temperatures (from left to right) $T=2.5,2.0,1.8$, 1.5 , and 1.0.

scenario in which the onset of the stretched exponential relaxation coincides with the percolation temperature $T_{p}$ $=3.817 \pm 0.005$ (see Sec. III).

We also simulated the FF Ising model on a square lattice of size $L=60$. We calculated the relaxation functions of the energy. Averages were made over 16 different random generator seeds, and between $10^{5}$ and $10^{6}$ steps for acquisition were taken, after about $10^{4}$ steps for thermalization.

In Fig. 5 we show the relaxation functions obtained for $T=2.5,2.0,1.8,1.5$, and 1.0. For all temperatures we fit the long time tail of the relaxation functions with Eq. (1).

The temperature dependence of exponents $\beta(T)$ is shown in Fig. 6. Note that $\beta(T)$ increases as function of $T$ from the value $\beta=0.61 \pm 0.05$ for $T=0.8$ to the value $\beta=1$ for $T \geqslant 2.0$. As we can see in Fig. 6, our estimate of the onset of the stretched exponential relaxation is also consistent, within the errors, with the percolation temperature $T_{p}=1.701$ [7]. Within the errors, the exponent $x(T)$ increases as function of $T$ from the value $x=0.4 \pm 0.2$ for $T=0.8$ to the value $x$ $=1.6 \pm 0.4$ for $T=2.5$.

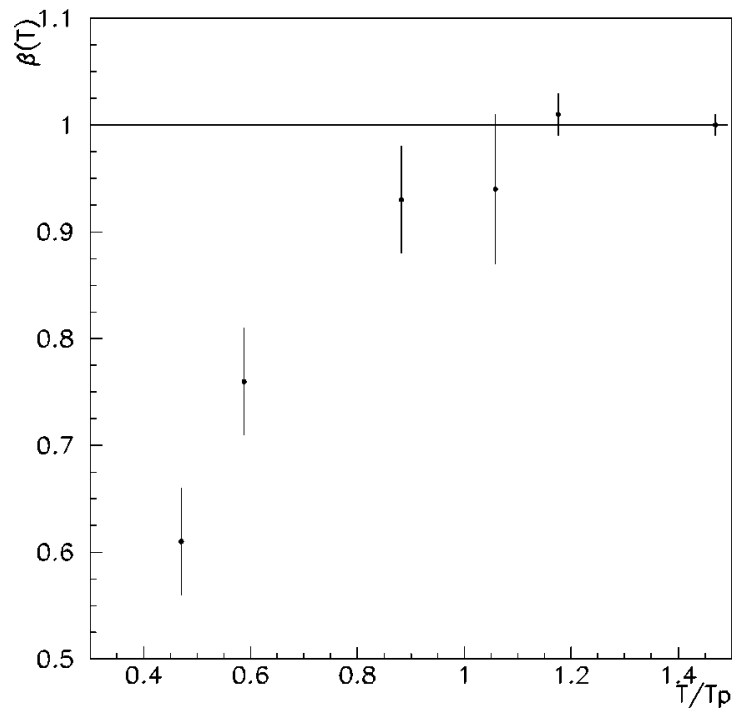

FIG. 6. Stretching exponents $\beta(T)$ as a function of $T / T_{p}$, the ratio of temperature over percolation temperature, for the $d=2 \mathrm{FF}$ Ising model, with spin flip dynamics and lattice size $L=60$.

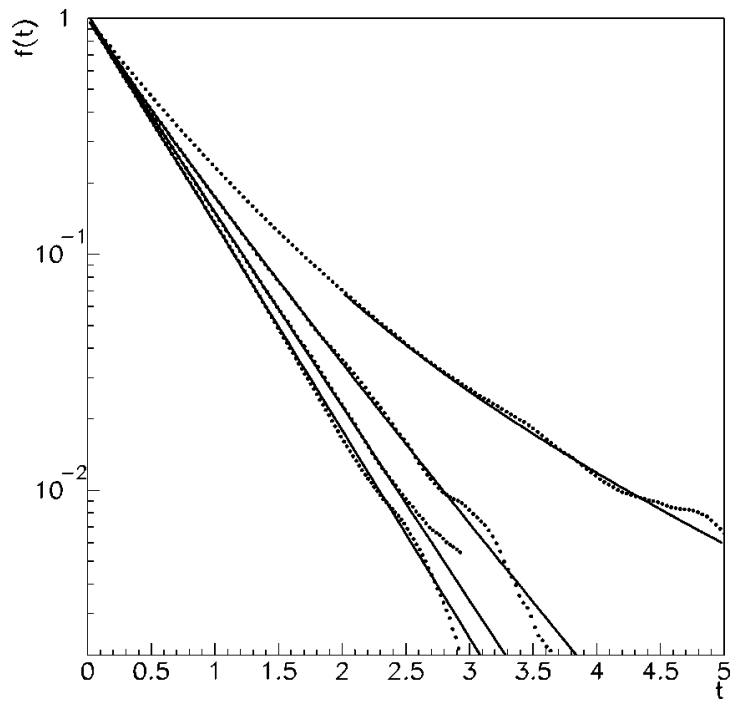

FIG. 7. Relaxation functions $f(t)$ of bond density as function of time $t$, for the $q=2 \mathrm{FF}$ bond percolation model, on a $d=3$ lattice of size $L=30$, for temperatures (from left to right) $T=4.0,3.5,3.0$, and 2.5 .

\section{RELAXATION FUNCTIONS OF THE " $q$-BOND FRUSTRATED PERCOLATION" MODEL}

In this section we analyze the dynamical behavior of the model defined by Eq. (4) with $q=2$, simulated by the bond flip dynamics [7]. The dynamics is carried out in the following way: at each step we choose at random a particular edge on the lattice; we calculate the probability $P$ of changing its state, that is, of creating a bond if the edge is empty, and of destroying the bond if the edge is occupied; and, finally, we change the state of the edge with probability $P$.

For each temperature $T, 16$ different runs were made, varying the random number generator seed, on a FF cubic lattice of size $L=30$. We took about $10^{3}$ steps for thermalization, and between $10^{4}$ and $10^{5}$ steps for acquisition, calculating at each step the density of bonds $\rho(t)$. The relaxation function of the density of bonds is defined as

$$
f(t)=\frac{\langle\delta \rho(t) \delta \rho(0)\rangle}{\left\langle(\delta \rho)^{2}\right\rangle},
$$

where $\delta \rho(t)=\rho(t)-\langle\rho\rangle$. For each value of $T$, we averaged the 16 functions calculated and evaluated the error as a standard deviation of the mean. We consider a unit of time to consist of $\mathcal{G}\langle\rho\rangle^{-1}$ single update trials, where $\mathcal{G}=3 L^{3}$ is the number of edges on the lattice.

In Fig. 7 we show the results obtained for temperatures $T=4.0,3.5,3.0$, and 2.5. For $T=4.0$ and 3.5 we fitted the calculated points with the function in Eq. (1).

The value of $\beta$ extracted from the fit is equal to one within the error, and the value of $x$ is zero. Thus for these temperatures the relaxation is purely exponential.

For $T<3.5$ we observe a two step decay, and only the long time regime of the relaxation functions could be fitted by Eq. (1). The value of $\beta$ extracted is less than 1, showing that stretched exponential relaxation has appeared for these temperatures. In Fig. 8 the values of $\beta(T)$ as function of the ratio $T / T_{p}$ are shown, with errors estimation. The exponent 


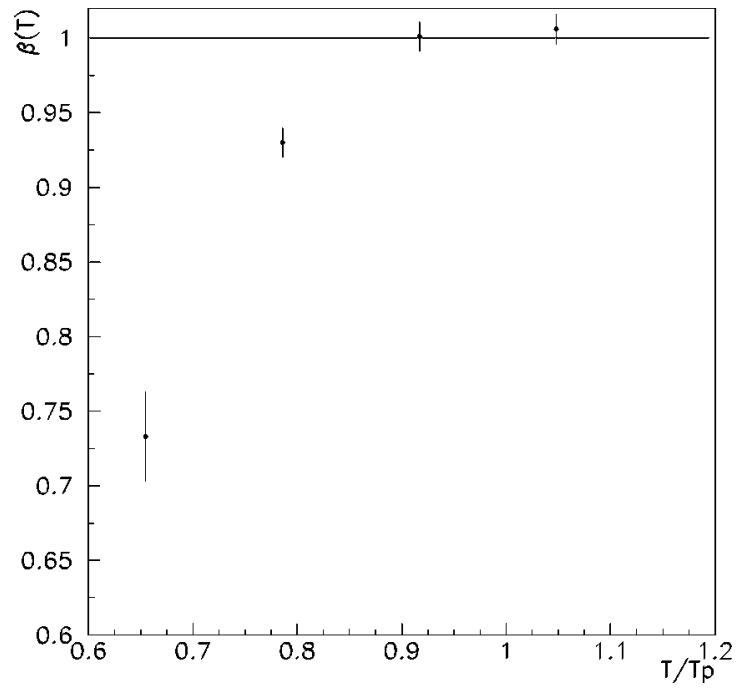

FIG. 8. Stretching exponents $\beta(T)$ as a function of $T / T_{p}$, the ratio of temperature over percolation temperature, for the $q=2 \mathrm{FF}$ bond percolation model, on a $d=3$ lattice of size $L=30$.

$x(T)$ becomes nonzero only for $T=2.5$, for this value of temperature we obtain $x=1.1 \pm 0.1$. As we can see in Fig. 8, our estimate of the onset of stretched exponential relaxation is consistent, within the errors, with the percolation temperature $T_{p}=3.817 \pm 0.005$ of the KFCK clusters.

\section{CONNECTION WITH THE RANDOM WALK PICTURE}

In this section we make a connection between our model and the random walk picture of Campbell et al. [9], which we will briefly illustrate. Consider an hypercube in a $D$-dimensional space. Each summit is occupied with a probability $p$. On such a dilute lattice, a random walker is allowed to diffuse, like the "ant' on a percolating cluster in the de Gennes picture. The mean square displacement after a time $t$ is given by

$$
r^{2}(t) \equiv \frac{\left\langle\sum_{i=1}^{D}\left(x_{i}(t)-x_{i}(0)\right)^{2}\right\rangle}{D},
$$

where $D$ is the hypercube dimension, $x$ is a $D$-dimensional vector of components 0 and 1 that identify the hypercube $2^{D}$ summits, and $x(t)$ indicates the "ant'" position at the time $t$.

Campbell et al. suggested in the Ising SG model that an accessible region in the space of configurations, compact at high temperature, becomes ramified at a temperature $T^{*}$, and that a complex space of configurations is responsible for the appearing of nonexponential relaxation. They also supposed that this temperature $T^{*}$ is the percolation temperature of the KFCK clusters. The idea is that the diffusive ant mimics quite well the evolution in the space of configurations in the SG model.

In the study of the random walk on a randomly occupied hypercube, they found that for $p \leqslant p^{*}$ the function $r^{2}(t)$ becomes nonexponential and is well approximated by a stretched exponential. But it is not possible to associate a value of temperature with this probability.
TABLE I. Fit parameters for the autocorrelation functions $f(t)$ calculated with bond flip dynamics for the $q=2$ bond FF percolation model.

\begin{tabular}{ccc}
\hline \hline$T$ & $\beta$ & $\tau^{\beta}$ \\
\hline 5.0 & $0.99 \pm 0.02$ & $0.16 \pm 0.01$ \\
3.5 & $1.00 \pm 0.02$ & $0.19 \pm 0.01$ \\
2.5 & $0.98 \pm 0.02$ & $0.24 \pm 0.01$ \\
2.0 & $0.98 \pm 0.02$ & $0.29 \pm 0.01$ \\
1.7 & $0.94 \pm 0.02$ & $0.44 \pm 0.01$ \\
1.3 & $0.75 \pm 0.02$ & $1.1 \pm 0.2$ \\
0.7 & $0.51 \pm 0.02$ & $2.8 \pm 0.2$ \\
\hline \hline
\end{tabular}

To make the connection between the bond frustrated percolation formalism and the random walk picture we introduce the local bond density autocorrelation function $f(t)$,

$$
f(t)=\frac{\sum_{i=1}^{\mathcal{G}}\left\langle b_{i}(t) b_{i}(0)\right\rangle-\left\langle b_{i}(t)\right\rangle^{2}}{\sum_{i=1}^{\mathcal{G}}\left\langle b_{i}(t)\right\rangle-\left\langle b_{i}(t)\right\rangle^{2}},
$$

where $\mathcal{G}=d L^{d}$ is the number of lattice edges, $d$ is the lattice dimension and $b_{i}=0$ if the $i$ th bond is missing and $b_{i}=1$ if the $i$ th bond is present. The variables $b_{i}(t)$ are the coordinates in the space of configurations of the $q$-bond FF percolation model, which evolve by bond flip dynamics. In analogy with the picture proposed by Campbell et al., these variables can be interpreted as the coordinates of a walk on the summits of a $\mathcal{G}$-dimensional hypercube. For a fixed value of the temperature the walk will be confined in the subspace with density of bonds corresponding to that temperature. (The walk in such subspace is not random, since each step has a weight, which is a function of the cluster number variation. If we consider the $q=1$ bond frustrated percolation model, the walk would be random.)

Due to frustration not all configurations are allowed, and therefore the walk occurs on a dilute space. By changing the temperature the space of configurations where the walk is confined changes, and therefore the density of allowed sites in such a region of the space of configurations also changes. This is realized in an artificial way in the picture proposed by Campbell et al., occupying randomly the hypercube summits (that represent the accessible states in the space of configurations). By changing the temperature, one may therefore reach a percolation threshold in the space of configurations. This would correspond to a breaking of the ergodicity. At higher temperature, however, the space of configurations may become ramified and stretched exponentials start to appear. Equation (12) can be related to the distance traveled by the random walk $r(t)$, via the relation $r^{2}(t)=2(\langle\rho\rangle-f(t))$.

We have simulated, by bond flip dynamics, the $q=2$ bond FF percolation model on a square lattice of size $L=60$. We have calculated the temperature dependence of the autocorrelation function in Eq. (12). We find an exponential relaxation at high temperatures, while for $T<T^{*}$ the long time behavior of relaxation functions becomes nonexponential and is well approximated by a stretched exponential. 


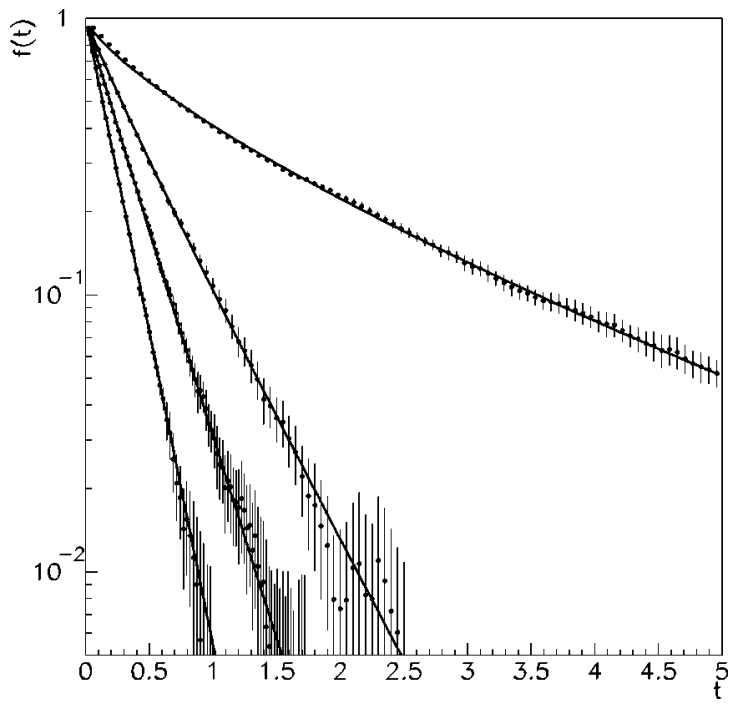

FIG. 9. Autocorrelation functions $f(t)$ as a function of time $t$ for the $q=2$ FF bond percolation model, on a $d=2$ lattice of size $L$ $=60$, for temperatures (from left to right) $T=3.5,2.0,1.7$, and 1.3.

In Table I we show the fit parameters. Our estimate of the onset of stretched exponential relaxation functions is also consistent, within the errors, with the percolation temperature $T_{p}=1.701$. In Fig. 9 we show the functions $f(t)$ for temperatures $T=3.5,2.0,1.7$, and 1.3, and in Fig. 10 the temperature dependence of the exponents $\beta(T)$ as a function of $T / T_{p}$.

We conclude that it is possible to apply the picture proposed by Campbell et al. to the $q$-bond frustrated percolation model. Furthermore our results are consistent with the hypothesis that the onset of nonexponential relaxation function coincides with $T_{p}$. Note that we cannot exclude numerically that stretched exponentials are present even at temperatures higher than the percolation transition, with an extremely small amplitude. This is also consistent with Campbell picture, where the crossover from compact to ramified structure in the space of configurations is not sharp.

\section{CONCLUSIONS}

In fully frustrated models we have numerically found an exponential relaxation above the percolation temperature $T_{p}$, while for $T<T_{p}$ the long time tail of the relaxation functions can be fitted with a stretched exponential in both 2D [7] and $3 \mathrm{D}$ systems. These results suggest that the percolation tran-

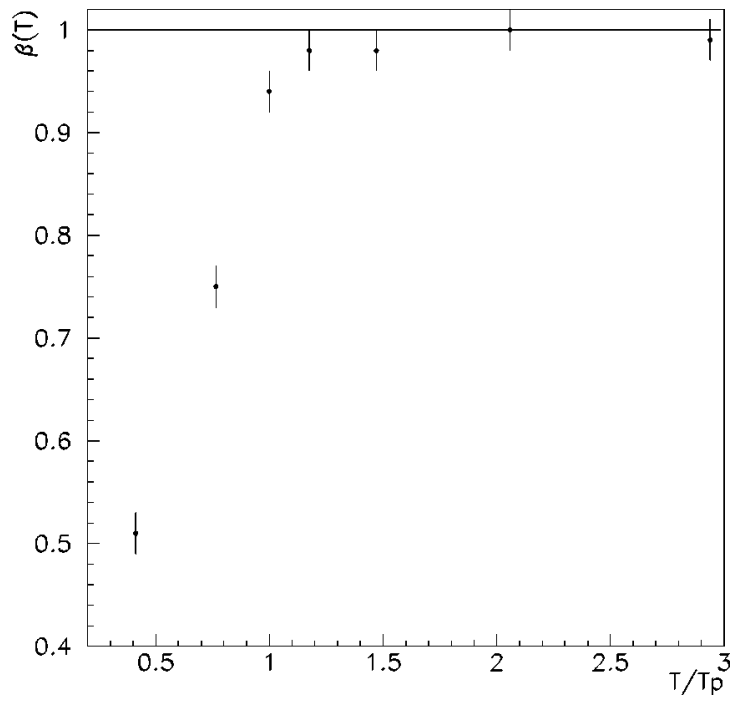

FIG. 10. Stretching exponents $\beta(T)$ as a function of $T / T_{p}$, the ratio of temperature over percolation temperature, for the $q=2 \mathrm{FF}$ bond percolation model, on a $d=2$ lattice of size $L=60$.

sition may play a role in the dynamical transition of frustrated systems without disorder. In particular, the role can be understood considering the physical meaning of the percolation transition in a generalization of the SG model (the $q$-state Potts SG [8]). We suggest that the percolation transition marks the appearing of the "large scale" effects of frustration. Below the percolation temperature, because of the presence of a spanning cluster, bond loops of any dimension may be closed, and therefore global effects of frustration are observed.

Note that in a previous paper [7] we also studied a model, "locally frustrated bond percolation," in which only frustrated loops whose length is equal to 4 are forbidden. The model exhibits the same critical properties as the random bond percolation, showing that this kind of frustration is "too local" to change the universality class of transition. Similarly, the relaxation functions in the long time regime can always be fitted with an exponential function.

\section{ACKNOWLEDGMENTS}

We would like to thank Chris Hanley for helpful comments. This work was supported in part by the European TMR Network-Fractals under Contract No. FMRXCT980183 and by MURST (PRIN-97). Simulations were done on the parallel Cray T3D at CINECA, Bologna.
[1] See, for example, F. Mezei and A. P. Murani, J. Magn. Magn. Mater. 14, 211 (1979); C. Meyer, F. Hartmann-Boutron, Y. Gros, and I. A. Campbell, ibid. 46, 254 (1985).

[2] W. L. McMillan, Phys. Rev. B 28, 5216 (1983).

[3] A. T. Ogielski, Phys. Rev. B 32, 7384 (1985).

[4] M. Randeria, J. P. Sethna, and R. G. Palmer, Phys. Rev. Lett. 54, 1321 (1985).
[5] F. Cesi, C. Maes, and F. Martinelli, Commun. Math. Phys. 188, 135 (1997).

[6] A. Fierro, A. de Candia, and A. Coniglio, Phys. Rev. E 56, 4990 (1997).

[7] G. Franzese and A. Coniglio, Phys. Rev. E 58, 2753 (1998).

[8] R. B. Griffiths, Phys. Rev. Lett. 23, 17 (1969).

[9] I. A. Campbell, J. M. Flesselles, R. Jullien, and R. Botet, J. 
Phys. C 20, L47 (1987); I. A. Campbell and L. W. Bernardi, Phys. Rev. B 50, 12643 (1994); I. A. Campbell, J. M. Flesselles, R. Jullien, and R. Botet, ibid. 37, 3825 (1988).

[10] S. Scarpetta, A. de Candia, and A. Coniglio, Phys. Rev. E 55, 4943 (1997).

[11] S. C. Glotzer and A. Coniglio, Comput. Mater. Sci. 4, 325 (1995).

[12] C. M. Fortuin and P. W. Kasteleyn, Physica (Amsterdam) 57, 536 (1972).

[13] A. Coniglio and W. Klein, J. Phys. A 12, 2775 (1980).

[14] J. Villain, J. Phys. C 10, 1717 (1977).

[15] A. Coniglio, F. di Liberto, G. Monroy, and F. Peruggi, Phys. Rev. B 44, 12605 (1991).
[16] V. Cataudella, A. Coniglio, L. de Arcangelis, and F. di Liberto, Physica A 192, 167 (1993); A. Coniglio, F. di Liberto, G. Monroy, and F. Peruggi, J. Phys. A 22, L837 (1989).

[17] F. Y. Wu, Rev. Mod. Phys. 54, 235 (1982).

[18] U. Pezzella and A. Coniglio, Physica A 237, 353 (1997).

[19] F. di Liberto and F. Peruggi, Physica A 248, 273 (1998).

[20] A. de Candia, V. Cataudella, and A. Coniglio (unpublished).

[21] H. T. Diep, P. Lallemand, and O. Nagai, J. Phys. C 18, 1067 (1985).

[22] R. H. Swendsen and J. S. Wang, Phys. Rev. Lett. 58, 86 (1987).

[23] D. Stauffer and A. Aharony, Introduction to Percolation Theory (Taylor \& Francis, London, 1994). 